\title{
The Hyperactivity/Inattention Subscale of the Strengths and Difficulties Questionnaire Predicts Short- and Long-Term Weight Loss in Overweight Children and Adolescents Treated as Outpatients
}

\author{
Andreas van Egmond-Froehlich ${ }^{a}$ Monika Bullinger ${ }^{b}$ Reinhard W. Holl ${ }^{c}$ \\ Ulrike Hoffmeister ${ }^{c}$ Reinhard Mann ${ }^{d}$ Cornelia Goldapp ${ }^{d}$ \\ Joachim Westenhoefer ${ }^{\mathrm{e}} \quad$ Ulrike Ravens-Sieberer ${ }^{f}$ Martina de Zwaan ${ }^{g}$ \\ ${ }^{a}$ Department of Pediatrics, Medical University of Vienna, Vienna, Austria, ${ }^{b}$ Department \\ for Medical Psychology, University Clinic Hamburg-Eppendorf, Hamburg, 'Department of \\ Epidemiology, Ulm University, Ulm, d Federal Center for Health Education (BZgA), Cologne, \\ e Department of Health Sciences, Hamburg University of Applied Sciences, ${ }^{f}$ Research Unit \\ Child Public Health, University Clinic Hamburg-Eppendorf, Hamburg, 9 Department of \\ Psychosomatic Medicine and Psychotherapy, Hanover Medical School, Hanover, Germany
}

\section{Key Words}

Overweight $\cdot$ Obesity $\cdot$ Children $\cdot$ Adolescents $\cdot$ Prediction $\cdot$ Hyperactivity $\cdot$ Attention deficit $\cdot$ Weight loss

\begin{abstract}
Objective: The success of treatment for pediatric obesity is variable and often unsatisfactory. This study elucidates the influence of inattention and hyperactivity/impulsivity on short- and long-term weight loss and maintenance after outpatient treatment. Methods: We included 8- to 16-year-old overweight and obese participants treated in 17 multidisciplinary outpatient treatment centers in a nationwide observational study. All treatment centers that reported long-term (1-year) follow-up weight data of at least $60 \%$ of the participants were included. At the beginning and end of treatment and at 1 year follow-up weight and height were measured at the center. Inattention and hyperactivity/impulsivity were assessed with the hyperactivity/ inattention subscale $(\mathrm{HI})$ of the parent-rated Strengths and Difficulty Questionnaire (SDQ). General linear models were used with the standard deviation scores of the BMI (BMI-SDS) as dependent variable and $\mathrm{HI}$ scores as main independent variable adjusting for age, sex, baseline BMI-SDS, and center. Results: 394 participants were included (57\% female, age: $11.7 \pm 2.0$ years, baseline BMI-SDS $\left.2.32 \pm .46 \mathrm{~kg} / \mathrm{m}^{2}\right)$. HI scores were significantly associated with short-
\end{abstract}


and long-term BMI-SDS ( $p<0.0005)$, with higher baseline HI scores predicting less weight loss. Conclusions: Our results indicate that inattention and hyperactivity/impulsivity are linearly associated with reduced short- and long-term weight loss. Implications for treatment are discussed.

Copyright (C) 2012 S. Karger GmbH, Freiburg

\section{Introduction}

Obesity and its consequences present a serious public health problem. The prevalence of obesity in German children and adolescents of currently $6.3 \%$ (defined by national standards) [1] has doubled in the past 15 years [2].

Combined behavioral lifestyle interventions reduce weight [3], but success is highly variable between patients, and long-term success is often unsatisfactory.

There have been numerous reports investigating a possible association of attentiondeficit/hyperactivity disorder (ADHD) or ADHD symptoms with obesity in children and adolescents that have been recently reviewed $[4,5]$.

Most were clinical studies [6-11] without adequate adjustments for putative confounding variables. Clinical samples of children with ADHD from inpatient psychiatric centers $[6,8$, $10,11]$ and of extremely obese children hospitalized in a tertiary university center for eating disorders [9] may exaggerate the putative association between ADHD and obesity. Two studies used historical reference data rather than a control group $[6,10]$ and did not account for the epidemic rise in the prevalence of obesity. Two studies utilizing neuropsychological tests $[7,12]$ showed higher impulsivity in obese compared to normal-weight children.

Among the few epidemiological studies, three found an association between a diagnosis of ADHD or high rates of ADHD symptoms and obesity [13-15] while three did not find any association between ADHD symptoms measured by the Strengths and Difficulties Questionnaire (SDQ) and obesity [16-18]. In a recent representative German community survey of 2,863 11- 17-year-old children and adolescents, overweight/obese minors were twice as likely to have an ADHD diagnosis, and youth with ADHD were twice as likely to be overweight or obese [15]. However, Egmond-Fröhlich et al. [18] reported that in a German population sample of 11,159 8- to 17-year-old children and adolescents the association between obesity and ADHD symptoms as measured with the hyperactivity/inattention (HI) subscale of the SDQ was no longer significant after adjusting for family background variables.

Longitudinal studies were also contradictory. One reported prediction of obesity by low self-regulation skills (emotion regulation, sustained attention, and inhibitory control/ reward sensitivity) [19], and one reported no prediction of obesity by ADHD symptoms [20].

Of more clinical relevance is the predictive effect of inattentive and hyperactive/ impulsive behavior on weight loss during and after treatment for obesity. In adults there is initial evidence that patients with ADHD are significantly less successful at losing weight compared to non-ADHD patients [21,22]. Regarding the influence of impulsivity and inhibitory control on treatment course in children and adolescents, the data of the few available studies are contradictory [23, 24]. Nederkoorn et al. [23] found in a small sample of 26 obese children that the most impulsive children lost less weight. Impulsivity was measured with the stop signal task [25], a neuropsychological task that evaluates the capacity to inhibit a pre-potent response provoked by an external cue. Pauli-Pott et al. [24] reported that a score derived by principal component analysis from both a Go /No Go and an interference task that was interpreted as representing impulsivity correlated significantly and positively with weight loss in a study involving 111 overweight and obese children and adolescents. 
ADHD, the most prevalent mental health disorder in childhood, is characterized by inattention, hyperactivity, and impulsivity. Questionnaire-based screening instruments such as the SDQ capture these behaviors. However, the association between the clinical diagnosis of ADHD, questionnaire scores, and neuropsychological tests that aim to capture aspects of impulsivity and inattention is limited. This reflects differences between laboratory and reallife settings, the complexity of the involved psychological constructs, and measurement error.

The present paper adds to the existing research by evaluating the predictive effect of inattention and HI (as assessed with the HI subscale of the parent-rated SDQ) on short- and long-term weight loss outcome in children and adolescents during and after outpatient weight loss treatment. To our knowledge, this is the first study prospectively investigating the predictive effect of combined inattentive impulsive and hyperactive behaviors on weight loss treatment success in a large sample of children and adolescents.

\section{Material and Methods}

\section{Participants and Procedures}

\section{Participants}

Out of 480 outpatient treatment facilities for pediatric overweight and obesity, 135 agreed to participate in a nationwide observational study initiated and financed by the Federal Center for Health Education (Bundeszentrale für gesundheitliche Aufklärung; $\mathrm{BZgA}$ ) of which 52 were randomly selected for participation [26]. The sample consisted of patients with a BMI above the 90th percentile seeking weight loss treatment between August 2005 and August 2006 [2]. Participants had to be between 8 and 16 years old. Weight and height were measured at each institution at baseline at the end of treatment and at 1-year follow-up. The data of treatment centers with a 1-year follow-up rate of less than $60 \%$ were excluded from the analyses to avoid participant bias. Thus, the average 1-year follow-up rate of the remaining 17 centers was $79 \%$ which approximates the original goal ( $80 \%)$ for the return rate in our observational study.

\section{Obesity Treatment}

The selected treatment centers were all offering multi-modal outpatient weight loss treatment for children and adolescents targeting both the children and their parents. Treatment was not experimentally influenced by a study protocol as the study was designed as an observational survey capturing the current status of obesity care in Germany. Therefore, intensity and focus varied between treatment centers. The characteristics of the outpatient treatment in the 17 treatment centers are described in table 1.

\section{Procedures}

At the beginning of the program, (t0) weight and height of the participants were measured at the treatment center. Participating patients and a parent were asked to complete questionnaires. Anthropometric measurements and questionnaires were repeated at the end of treatment ( $\mathrm{t} 1$ ) and at the 1-year follow-up assessment (t2). Follow-up measurements that were performed more than 18 month after the end of the treatment phase were not included into the analyses. Written informed consent was obtained from the participants and their parents. The study was approved by the local ethics committee of the University of Ulm.

\section{Assessment Instruments}

$B M I-S D S$

BMI $\left(\mathrm{kg} / \mathrm{m}^{2}\right)$ was calculated from measured weight and height. Standardized BMI (BMI-SDS) was computed using the LMS method [27] employing national age- and sex-specific norms [2].

\section{Sociodemographic Variables}

In the parent questionnaire maternal and paternal education, profession, employment status, as well as family income were recorded [28]. A socioeconomic status score (SES) was calculated using an algorithm from the recent German Health Interview and Examination Survey for Children and Adolescents (KIGGS) [28]. 
Table 1. Characteristics of the treatment in the included 17 outpatient treatment centers

\begin{tabular}{|c|c|c|c|c|c|c|c|c|c|c|c|c|c|c|c|c|c|}
\hline & \multicolumn{17}{|c|}{ Center } \\
\hline & 1 & 2 & 3 & 4 & 5 & 6 & 7 & 8 & 9 & 10 & 11 & 12 & 13 & 14 & 15 & 16 & 17 \\
\hline Sample size & 8 & 14 & 37 & 33 & 36 & 41 & 22 & 23 & 15 & 17 & 5 & 32 & 49 & 19 & 10 & 10 & 8 \\
\hline Treatment duration, months & 10 & 22 & 14 & 11 & 17 & 11 & 11 & 12 & 5 & 14 & 12 & 8 & 12 & 14 & 7 & 13 & 12 \\
\hline Total training units & 167 & 13 & 10 & 149 & 201 & 11 & 240 & 38 & 48 & 10 & 85 & 77 & 164 & 12 & 7 & 50 & 2 \\
\hline Parent training $\$$ & 38 & 6 & 5 & 19 & 55 & 5 & 75 & 10 & 4 & 4 & 8 & 28 & 24 & 3 & 2 & 9 & 2 \\
\hline Patient training $\$$ & 129 & 7 & 5 & 130 & 146 & 6 & 195 & 30 & 43 & 6 & 80 & 52 & 140 & 9 & 5 & 41 & 6 \\
\hline Psychosocial $^{\$}$ & 30 & 4 & 0 & 16 & 30 & 1 & 20 & 8 & 14 & 1 & 6 & 9 & 16 & 2 & 1 & 4 & 0 \\
\hline Dietary counseling $\$$ & 6 & 2 & 3 & 12 & 26 & 2 & 20 & 4 & 14 & 1 & 36 & 35 & 60 & 3 & 3 & 4 & 10 \\
\hline Physical activity ${ }^{\$}$ & 91 & 1 & 0 & 100 & 82 & 2 & 120 & 14 & 14 & 2 & 36 & 8 & 60 & 4 & 1 & 32 & 20 \\
\hline Medical psychoeducation $\$$ & 2 & 0 & 2 & 2 & 8 & 1 & 5 & 2 & 1 & 1 & 0 & 1.5 & 4 & 0 & 0 & 1 & 0 \\
\hline Drop-outs & 0 & 0 & 19 & 3 & 3 & 5 & 4 & 12 & 0 & 0 & 0 & 9 & 6 & 24 & 0 & 0 & 12 \\
\hline Loss-to follow-up, $\%$ & 12 & 14 & 35 & 6 & 3 & 23 & 22 & 39 & 33 & 24 & 20 & 9 & 30 & 24 & 30 & 0 & 12 \\
\hline
\end{tabular}

\$The duration is reported in units of $45 \mathrm{~min}$.

Parents were asked to complete the 25-item SDQ which assesses mental health problems in children and adolescents aged 4-16 years. Five subscales consisting of 5 items each can be calculated: emotional symptoms, conduct problems, HI, peer relationship problems, and prosocial behavior. Each of the items was rated by one parent as 'not correct', 'partly correct', or 'completely correct'. Each subscale has possible values from 0 to 10 . Its reliability and validity make the SDQ a useful brief measure of psychological attributes $[29,30]$. The internal consistencies of the SDQ subscales in our sample were generally satisfactory and very similar to the results of other groups [29] with Cronbach's $\alpha$ of 0.74 (emotional symptoms), 0.58 (conduct problems), 0.75 (HI), 0.71 (peer relationship problems), and 0.68 (pro-social behavior).

We were primarily interested in the HI subscale. The 5 items of the HI subscale were originally selected to tap inattention ( 2 items), hyperactivity ( 2 items), and impulsiveness ( 1 item) because these are the three key symptom domains for a diagnosis of ADHD according to DSM IV [31] criteria. Using clinical interviews as a gold standard the SDQ outperformed the Child Behavior Checklist (CBCL) in detecting inattention and hyperactivity [32]. The parent rated SDQ was also used in the KIGGS study [33], a large survey for children and adolescents in Germany; hence, recent national reference values are available. Of the 8,857 KIGGS participants within the age range of our study, $85.6 \%$ had normal, $6.0 \%$ borderline, and $8.4 \%$ abnormal HI scores with a median score of 3 (interquartile range 1-5). As in the KIGGS study, we used the age-dependent cut-off values for borderline and abnormal scores from the English normative data [34]. The SDQ total difficulties score is formed by summing the results from all the scales except the prosocial scale.

\section{Patient Self-Report Questionnaire}

The Eating Questionnaire for Children (EQC) [35] is an adaptation for children of the German version [36] of the Three Factor Eating Questionnaire [37]. This well-validated questionnaire consists of 16 items and yields two main factors: disinhibition of eating behavior and cognitive control (dietary restraint) each consisting of 8 items (in our sample Cronbach's $\alpha$ were 0.73 and 0.70 , respectively).

\section{Statistical Analysis}

All analyses were performed using SPSS (version 17.0 for Windows; SPSS, Inc., Chicago, IL, USA). First, participants with and without measured 1-year follow-up weight and height data were compared using Mann-Whitney U tests for continuous variables and chi-square tests for sex.

The primary research question concerned the predictive effect of the HI subscale score at baseline (t0) on short-term (end of treatment, t1) and long-term (1-year follow-up, t2) weight loss. Thus, BMI-SDS 
values at $\mathrm{t} 1$ or $\mathrm{t} 2$ were entered as the dependent variables and baseline ( $\mathrm{t} 0$ ) BMI-SDS as covariate in general linear models (GLM; SPSS 17.1). In addition, the prediction of BMI-SDS change during the maintenance phase ( $\mathrm{t} 1-\mathrm{t} 2$ ) was analyzed by entering BMI-SDS at $\mathrm{t} 2$ as the dependent and BMI-SDS at $\mathrm{t} 1$ as covariate into the GLM.

All analyses were adjusted for age, sex, and treatment center.

We conducted an intention-to-treat analysis by applying the last observation carried forward method for missing $\mathrm{t} 1$ and $\mathrm{t} 2 \mathrm{BMI}$-SDS values. Also in accordance with the proposed strategy for intention-to-treat analysis [38], we performed a sensitivity analysis regarding the assumptions involving missing data, defining realistic upper and lower limits of weight-related outcomes in patients lost to follow-up. A complete case analysis optimistically assumes that loss to follow-up is completely random, while a worst case scenario assumes that BMI increases by 1 SD from the last observation.

In addition to the SDQ-HI subscale, other potential predictors known from the literature for which measurement instruments were available in our survey were entered as independent variables in univariate GLMs. These included the conduct problem subscale of the SDQ [39], the eating disinhibition subscale of the EQC [40, 41], and maternal BMI [42-44]. All significant predictors were then entered together in a multivariate GLM (adjusted as above). The model was checked for normal distribution and homoscedasticity of the dependent variables.

Additional explorative predictor analyses were performed with the SES, the remaining SDQ subscales, the SDQ total difficulties score, and the cognitive control subscale of the EQC.

We also performed a binary logistic regression with the short- and long-term treatment success as the dependent variable and the same independent variables and covariates as described above. Treatment success was defined as a reduction of BMI-SDS of at least 0.2 . This cut-off was recommended by a commission on patient education programs for overweight and obese children [45] because it corresponds to a weight reduction of $5 \%$, which is used to define success of weight reduction and maintenance in adults.

Interaction terms between HI scores and sex, age, BMI-SDS at baseline, eating disinhibition, and SES were added to the GLM model in addition to the main terms in order to assess moderators for a possible predictive effect of HI scores on BMI-SDS.

\section{Results}

\section{Sample Characteristics}

In the selected 17 centers, 394 participants fulfilled the inclusion criteria. HI subscale scores at baseline were available in 389 participants (98.7\%). 26 patients (6.9\%) dropped out during treatment (table 1). Measured weight and height data at the end of treatment were available in 379 participants (96\%). Measured weight and height data at 1-year followup were available in 325 participants. However, in 13 cases the follow-up assessment was conducted more than 18 months after $\mathrm{t} 1$; therefore, the data were excluded, reducing the number of available BMI values at follow-up to 312 (79\%).

Participants with missing weight and height data at 1-year follow-up had significantly higher HI scores at baseline ( $4.23 \pm 2.30$ vs. $3.53 \pm 2.26, \mathrm{p}=0.013, \mathrm{U}=10,150, \mathrm{n} 1=80, \mathrm{n} 2$ $=309)$, a significantly shorter treatment duration $(0.81 \pm 0.42$ vs. $1.03 \pm 0.32$ years, $\mathrm{p}<0.0005, \mathrm{U}=6,992, \mathrm{n} 1=67, \mathrm{n} 2=312$ ), and a trend towards a poorer short-term change in BMI-SDS (-0.160 \pm 0.312 vs. $-0.232 \pm 0.339, \mathrm{p}=0.063, \mathrm{U}=8,940, \mathrm{n} 1=67, \mathrm{n} 2=312)$ compared to participants with available weight and height data at follow-up. There were no significant differences regarding age $(p=0.68)$, BMI-SDS at baseline $(p=0.91)$, SES $(p=0.68)$, or sex $(p=0.26)$ between participants with and without available 1-year followup data.

The characteristics of the participants are given in table 2 . 
Table 2. Sample characteristics

n

Sex: male / female

Age $^{a}$, years

$\mathrm{BMI}^{\mathrm{a}}, \mathrm{kg} / \mathrm{m}^{2}$

BMI-SDS at baseline ${ }^{\mathrm{a}}$

Weight status (AGA reference) t0

Overweight (BMI $>90$ th percentile, $\leq 97$ th percentile)

Obesity (BMI $>97$ th percentile, $\leq 99.5$ th percentile)

Morbid obesity (BMI $>99.5$ th percentile)

Hyperactivity/inattention (HI) ${ }^{b}$

Normal

Borderline

Pathological

Socioeconomic status score ${ }^{b}$

High

Average

Low

Treatment duration ${ }^{\mathrm{a}}$, months

BMI-SDS at end of treatment $(\mathrm{t} 1)^{\mathrm{a}, \mathrm{c}}$

Weight status at end of treatment

Normal-weight (BMI $\leq$ p90th percentile)

Overweight (BMI $>90$ th percentile, $\leq 97$ th percentile)

Obesity (BMI $>97$ th percentile, $\leq 99.5$ th percentile)

Morbid obesity (BMI $>99.5$ th percentile)

Missing data

Follow-up duration ( $\mathrm{t} 2-\mathrm{t} 0)^{\mathrm{a}}$

BMI-SDS at follow up (t2) $)^{\mathrm{a}, \mathrm{c}}$

Weight status at follow-up (t2)

Normal-weight (BMI $\leq 90$ th percentile)

Overweight (BMI $>90$ th percentile, $\leq 97$ th percentile)

Obesity (BMI $>97$ th percentile, $\leq 99.5$ th percentile)

Morbid obesity (BMI $>99.5$ th percentile)

Missing data

Short-term change in BMI-SDS ${ }^{\text {a }}$

Long-term change in BMI-SDS ${ }^{\text {a }}$

$\%$ BMI-SDS reduction $\geq 0.2$ (missing data coded as failure)

Short-term

Long-term (1 year) (c) 2012 S. Karger GmbH, Freiburg www.karger.com/ofa

van Egmond-Froehlich et al.: The Hyperactivity/Inattention Subscale of the Overweight Children and Adolescents Treated as Outpatients

${ }^{\mathrm{a} C e l l}$ entries represent mean and standard deviation.

${ }^{\mathrm{b}}$ Cell entries represent median and interquartiles.

${ }^{\mathrm{c}}$ Return to baseline method for missing data.

\section{Prediction of Treatment Success}

\section{Prediction of Short- and Long-Term Weight Loss}

Short- and long-term BMI-SDS were significantly predicted by the HI subscale scores $(\mathrm{p}<0.0005)$ (table 3 and table 4). Long-term BMI-SDS was also associated with the conduct problem subscale scores of the SDQ and the eating disinhibition subscale score of the EQC at baseline, but not with maternal BMI. In the multivariate GLM only the HI score $(p<0.0005)$ remained an independent significant predictor of the 1-year BMI-SDS. 
Table 3. Prediction of short-term BMI-SDS reduction by inattention and hyperactivity/impulsivity and other relevant psychosocial variables ${ }^{\$}$

\begin{tabular}{lllllll}
\hline & \multicolumn{2}{l}{ Scale } & & & \\
\cline { 2 - 7 } & $\mathrm{B}^{\S}$ & $95 \% \mathrm{CI}$ & $\mathrm{N}$ & $\mathrm{F}$ & partial eta $^{2} \mathrm{p}$ \\
\hline Univariate analysis & & & & & & \\
$\quad$ SDQ HI scale & 0.035 & $0.021-0.049$ & 389 & 23.7 & 0.060 & $<0.0005$ \\
$\quad$ SDQ conduct problem scale & 0.010 & -0.008 to 0.028 & 389 & 1.2 & 0.003 & 0.277 \\
$\quad$ EQC eating disinhibition scale & 0.104 & -0.027 to 0.234 & 381 & 2.5 & 0.007 & 0.118 \\
$\quad$ Maternal BMI (self-report) & -0.001 & -0.005 to 0.006 & 371 & 0.1 & 0.000 & 0.772 \\
\hline
\end{tabular}

\$All using GLM with BMI-SDS at the end of treatment ( $\mathrm{t} 1$ ) as dependent variable and BMI-SDS at baseline (t0), sex and age as covariates and center as random variable.

§Per unit of the scales except for maternal BMI $\left(\mathrm{kg} / \mathrm{m}^{2}\right)$.

Table 4. Prediction of long-term BMI-SDS reduction by inattention and hyperactivity/impulsivity and other relevant psychosocial variables $\$$

\begin{tabular}{|c|c|c|c|c|c|c|}
\hline & \multicolumn{6}{|l|}{ Scale } \\
\hline & $\mathrm{B}^{\S}$ & $95 \% \mathrm{CI}$ & $\mathrm{N}$ & $\mathrm{F}$ & partial eta ${ }^{2}$ & $\mathrm{P}$ \\
\hline \multicolumn{7}{|l|}{ Univariate analysis } \\
\hline SDQ HI scale & 0.049 & $0.029-0.068$ & 389 & 23.5 & 0.060 & $<0.0005$ \\
\hline SDQ conduct problem scale & 0.028 & $0.003-0.053$ & 389 & 4.7 & 0.013 & 0.030 \\
\hline EQC eating disinhibition scale & 0.236 & $0.055-0.416$ & 381 & 6.6 & 0.018 & 0.011 \\
\hline Maternal BMI (self-report) & -0.005 & -0.012 to 0.003 & 371 & 1.5 & 0.004 & 0.217 \\
\hline Multivariate analysis (final model) & & & 378 & & & \\
\hline SDQ HI scale & 0.047 & $0.025-0.070$ & & 17.7 & 0.048 & $<0.0005$ \\
\hline SDQ conduct problem scale & -0.003 & -0.031 to 0.025 & & 0.0 & 0.000 & 0.860 \\
\hline EQC eating disinhibition scale & 0.169 & $0.015-0.353$ & & 3.2 & 0.009 & 0.072 \\
\hline
\end{tabular}

${ }^{\$}$ All using GLM with BMI-SDS at 1-year follow-up (t2) as dependent variable and BMI-SDS at baseline, sex and age as covariates and center as random variable.

§Per unit of the scales except for maternal BMI $\left(\mathrm{kg} / \mathrm{m}^{2}\right)$.

Additional exploratory analyses did not reveal an association between short- or longterm BMI-SDS and the baseline scores of the SDQ subscales emotional problems $(p=0.92$ and $p=0.96)$, peer problems $(p=0.57$ and $p=0.61)$, and prosocial behavior $(p=0.49$ and $p=0.28)$ as well as the cognitive control subscale of the EQC ( $p=0.57$ and $p=0.88)$, and the socioeconomic status $(\mathrm{p}=0.80$ and $\mathrm{p}=0.12)$ adjusting for age, sex, and initial BMI-SDS. The SDQ total difficulties score was significantly associated with both short- $(\mathrm{p}=0.030)$ and long-term ( $p=0.034$ ) weight loss; however, the associations were weaker ( $\operatorname{eta}^{2} 0.012$ and 0.012 , respectively) than the associations achieved with the SDQ HI scale alone ( $\operatorname{eta}^{2} 0.053$ and 0.054 , respectively).

Accordingly, using binary logistic regression analyses, patients with higher HI scores exhibited poorer short- and long-term treatment success rates defined as a BMI-SDS reduction of at least 0.2 . The OR $(95 \% \mathrm{CI})$ was $0.811(0.727-0.905)(\mathrm{p}<0.0005$, Wald $=14.0$, $\mathrm{N}=375)$ for the short-term success and $0.795(0.711-0.888)(\mathrm{p}<0.0005$, Wald $16.3, \mathrm{~N}=375)$ for the long-term success. 
Table 5. Prediction of weight loss maintenance by inattention and hyperactivity/impulsivity and other relevant psychosocial variables

\begin{tabular}{lllllll}
\hline Scale & $\mathrm{B}^{\S}$ & $95 \% \mathrm{CI}$ & $\mathrm{N}$ & $\mathrm{F}$ & Partial eta $^{2}$ & $\mathrm{p}$ \\
\hline Univariate analysis & & & & & & \\
$\quad$ SDQ HI scale & 0.015 & -0.001 to 0.031 & 374 & 3.6 & 0.010 & 0.060 \\
$\quad$ SDQ conduct problem scale & 0.019 & -0.001 to 0.039 & 374 & 3.4 & 0.010 & 0.065 \\
$\quad$ EQC eating disinhibition scale & 0.134 & -0.008 to 0.276 & 367 & 3.4 & 0.010 & 0.064 \\
$\quad$ Maternal BMI (self-report) & -0.005 & -0.011 to -0.001 & 358 & 3.0 & 0.009 & 0.085 \\
\hline
\end{tabular}

\$All using GLM with BMI-SDS at 1-year follow-up (t2) as dependent variable and BMI-SDS at the end of treatment ( $\mathrm{t} 1$ ), sex and age as covariates and center as random variable.

§er unit of the scales except for maternal BMI $\left(\mathrm{kg} / \mathrm{m}^{2}\right)$.

\section{Prediction of Weight Loss Maintenance}

When focusing specifically on the maintenance phase from the end of treatment ( $t 1)$ to the 1-year follow-up (t2), the BMI-SDS change was not significantly predicted by the HI scores at baseline (table 5). However, eating disinhibition at baseline and maternal BMI turned out to be marginally significant predictors of weight loss maintenance.

\section{Linearity}

The association between baseline HI scores and both short- and long-term BMI-SDS was linear as a quadratic term was rejected. HI scores remained a significant short- $(\mathrm{N}=315$, $\mathrm{B}=0.037(0.012-0.061), \mathrm{F}=8.3$, eta $\left.^{2}=0.029, \mathrm{p}=0.003\right)$ and long-term univariate predictor $\left(\mathrm{N}=315, \mathrm{~B}=0.061(0.027-0.095), \mathrm{F}=12.4\right.$ eta $\left.^{2}=0.041, \mathrm{p}<0.0005\right)$ even after excluding patients with borderline or pathological HI scores.

\section{Interactions with the Predictive Effect of HI Subscale Scores}

There was an interaction between HI subscale scores and age for the short- $(\mathrm{F}=4.8$, eta $\left.^{2}=0.013, \mathrm{p}=0.028\right)$ and long-term $\left(\mathrm{F}=2.8\right.$, eta $\left.^{2}=0.007, \mathrm{p}=0.097\right)$ prediction of BMI-SDS with a marginally stronger predictive effect in older participants. Neither the short- nor the long-term prediction of BMI-SDS by the HI subscale scores was moderated by sex ( $p=0.29$ and $p=0.51)$, BMI-SDS at baseline $(p=0.49$ and $p=0.63)$, SES $(p=0.76$ and $p=0.29)$, eating disinhibition $(\mathrm{p}=0.90$ and $\mathrm{p}=0.52)$, or treatment duration $(\mathrm{p}=0.47$ and $\mathrm{p}=0.99)$.

Results were virtually unchanged in complete case analyses.

Results were also virtually unchanged when missing BMI-SDS values at follow up were set to BMI-SDS at last observation +1 (a worsening by 1 SD after treatment corresponds to the 7.5th percentile of long-term BMI-SDS changes in completers).

\section{Discussion}

According to our expectation both short- and long-term weight loss success was negatively predicted by inattention and hyperactivity/impulsivity as measured with the $\mathrm{HI}$ subscale of the parent-rated SDQ.

The negative association between weight loss success and inattention as well as hyperactivity/impulsivity is in line with the results of some previous studies in children [12,23] and adults [46]. The contradictory results in one study that matched ours in age range and 
van Egmond-Froehlich et al.: The Hyperactivity/Inattention Subscale of the

Strengths and Difficulties Questionnaire Predicts Short- and Long-Term Weight Loss in Overweight Children and Adolescents Treated as Outpatients

treatment setting [24] might be explained by a different treatment regimen (emphasis on stimulus control and goal setting including behavior plans), differing instruments (Go-NoGo and interference tasks), or the statistical methods (principal component analysis to reduce dimensions). Unraveling this discrepancy may prove rewarding for research. As mentioned in the introduction, parent-rated symptoms of inattention and hyperactivity/impulsivity and neuropsychological tests measure different though pathophysiologically related aspects.

One explanation for the negative association between weight loss and inattention as well as hyperactivity/impulsivity is that attention and inhibitory control are required to resist overeating in an obesogenic environment $[12,23]$. In other words, individuals with high inattention and hyperactivity/impulsivity may exhibit eating patterns that may result not only in a higher BMI but might also interfere with the ability to intentionally reduce weight and to maintain it. The predictive effect of inattention and hyperactivity/impulsivity during the treatment phase indicates that attention and inhibitory control are required for changing behavior during behavioral obesity treatment.

The effect of inattention and hyperactivity/impulsivity increased somewhat with age. The control over food and beverage selection, media use, and physical activity in children is to a significant part in the hands of the parents. Accordingly, parent training for obesity proved to be at least as effective as combined parent and child training [47]. Adolescents on the other hand have more independent control over these behaviors and are thus to a larger extent responsible for the lifestyle changes during obesity treatment. Thus, treatment success should become more susceptible to moderation of behavior change by inattention and hyperactivity/impulsivity.

Eating disinhibition also predicted long-term weight change which is in line with the results of prospective non-interventional studies [40,41].

There was no significant interaction between eating disinhibition and HI scale scores regarding short- and long-term weight loss. In the multivariate model the long-term predictive effect of eating disinhibition (but not $\mathrm{HI}$ ) was weakened and became marginal indicating that the predictive effect of eating disinhibition may in part be based on its correlation with inattention and hyperactivity/impulsivity.

Self-reported maternal BMI was not associated with short- or long-term success. Results concerning the role of maternal BMI on weight loss success are contradictory. Some studies showed that children without a parental history of obesity achieved greater reductions in BMI-SDS [42-44], others did not find this association [48, 49].

The SDQ conduct problem scale did not emerge as an independent predictor of shortand long-term success.

Reflecting the comorbidity between ADHD, conduct disorders and emotional disorders [29], we found a significant correlation between the SDQ subscales HI, conduct problems, and emotional problems in our sample at baseline; however, only the HI subscale independently predicted weight change. This is of interest as depressive symptoms (related to the SDQ emotional problem scale) have been shown to reduce the treatment effect in obese children [50].

Finally, confirming previous reports $[42,51]$, SES was no predictor of treatment success.

\section{Strengths and Limitations of the Study}

The strengths of the study lie in its large sample size and the inclusion of 17 treatment facilities differing in treatment focus and intensity. In addition, the longitudinal design allows differentiating the influence of the predictive factors on short- and long-term weight 
loss and weight loss maintenance. The study employed measured weight and height data instead of self-reported data.

We had an acceptable rate of missing weight data at 1-year follow-up (21\%). Patients without 1-year follow-up data had higher inattention and hyperactivity/impulsivity at baseline and a trend towards less weight loss at the end of treatment. Also attrition is likely related to poorer outcome. The treatment of missing values may thus affect the correlation between $\mathrm{HI}$ and weight-loss. We showed however, that the results are insensitive to the assumptions regarding missing data, ranging from the exceedingly pessimistic BMI at last observation +1 SD, through the slightly optimistic last observation carried forward method, to the optimistic complete case analysis. Otherwise, there were no significant differences between participants with and without available weight data at the 1-year follow-up.

Clinician-made diagnosis of ADHD and psychoactive medications were not recorded. However, the HI scores predicted weight loss success also in the normal range of the scale. The association between HI scores and weight loss is thus not only based on patients with a full diagnosis of ADHD, who are expected to have pathological HI scores.

For practical reasons neither teacher-rated SDQ scales nor self-rated SDQ scales (for participants 11 years and above) were used in this study. These data would have improved the objectiveness of the assessment of ADHD symptoms in the patients [52,53] and added information on behavior in different environments.

Nederkoorn et al. [12, 23] achieved a fourfold partial eta ${ }^{2}(0.24)$ with the stop-signal task compared to our study but the economical SDQ questionnaire permitted its use in our much larger sample.

We were unable to unravel the behavioral mechanisms behind the prediction of weight change by the SDQ-HI scale. Eating behavior, physical activity, and media use were measured longitudinally by questionnaire in the current study. The changes of these behavioral variables did, however, not correlate with weight changes (data not shown), and we were thus unable to perform a path analysis. We think that the response to the used self-report instruments might have been biased by strong social desirability in obese children motivated for change and that objective measurements would be required.

We selected a subsample of outpatient treatment centers with acceptable return rates for our analysis. While there was no difference in baseline patient characteristics (age, sex, BMI-SDS, HI, SES etc.) between outpatient centers with more or less than $60 \%$ return rate at $\mathrm{t} 2$, they differed in the rate of treatment drop-outs, duration and intensity of treatment, and short-term treatment success. Although not representative for pediatric outpatient treatment in Germany, we see no reason why the prediction by psychosocial variables should be qualitatively different in these centers. Our results cannot be generalized to inpatient settings.

\section{Conclusions}

Since inattention and hyperactivity/impulsivity were shown to be negatively associated with short- and long-term weight loss success, treatment strategies should also focus on these issues. In view of the major impact of impulsivity, inhibitory control [12, 23], and sustained attention, preventive and therapeutic methods targeting these issues should be developed, integrated, and evaluated in training programs for obese children. Alternatively, the need for inhibitory control could be reduced by environmental control measures on a micro- (e.g. limited purchase of unhealthy food and presentation of fruit and vegetables by the parents), meso- (e.g. healthy food sales in school cafeterias and water fountains), and macro-level (e.g. legal restrictions on advertisement for energy-dense food directed at children and taxation of sugar-sweetened beverages). 
Future studies should investigate the moderation of the effects of obesity treatment by all facets of attention and inhibitory control and the related construct of self-regulation. They should also assess brain function (e.g. prefrontal/anterior cingulated cortex) and measure behavior objectively.

It should also be investigated whether the cross-sectional association between inattention/impulsivity and overweight/obesity that has been reported by the majority of the published studies could be mediated by the differing success rates in treatment (as confirmed in the current study) as these might extend to self-treatment. If this hypothesis was true, the cross-sectional correlation should be strongest in adolescent females who are most prone to diet.

\section{Acknowledgments}

This study was funded by the Federal Center for Health Education (Cologne, Germany), an agency of the German Federal Ministry for Health. We thank the participants and the participating obesity treatment centers. Furthermore we would like to thank Peter Bauer for reviewing the manuscript.

\section{Disclosure Statement}

The authors have no conflict of interest to declare.

\section{References}

1 Kurth BM, Schaffrath Rosario A: The prevalence of overweight and obese children and adolescents living in Germany. Results of the German Health Interview and Examination Survey for Children and Adolescents (KiGGS) (in German). Bundesgesundheitsblatt Gesundheitsforschung Gesundheitsschutz 2007;50:736743.

2 Kromeyer-Hauschild K, Wabitsch M, Geller F, Ziegler A, Geiß HC, Hesse V, von Hippel A, Jaeger U, Johnsen D, Kiess W, Korte W, Kunze D, Menner K, Müller M, Niemann-Pilatus A, Remer T, Schaefer F, Wittchen HU, Zabransky S, Zellner K, Hebebrand J: Perzentile für den Body Mass Index für das Kindes- und Jugendalter unter Heranziehung verschiedener deutscher Stichproben. Monatschr Kinderheilkd 2001;149:807-818.

3 Oude Luttikhuis H, Baur L, Jansen H, Shrewsbury VA, O’Malley C, Stolk RP, Summerbell CD: Interventions for treating obesity in children. Cochrane Database Syst Rev 2009:CD001872.

- 4 Cortese S, Angriman M, Maffeis C, Isnard P, Konofal E, Lecendreux M, Purper-Ouakil D, Vincenzi B, Bernardina BD, Mouren MC: Attention-deficit/hyperactivity disorder (ADHD) and obesity: a systematic review of the literature. Crit Rev Food Sci Nutr 2008;48:524-537.

5 Cortese S, Vincenzi B: Obesity and ADHD: clinical and neurobiological implications. Curr Top Behav Neurosci 2012;9:199-218..

6 Holtkamp K, Konrad K, Muller B, Heussen N, Herpertz S, Herpertz-Dahlmann B, Hebebrand J: Overweight and obesity in children with attention-deficit/hyperactivity disorder. Int J Obes Relat Metab Disord 2004; 28:685-689.

7 Braet C, Claus L, Verbeken S, Van Vlierberghe L: Impulsivity in overweight children. Eur Child Adolesc Psychiatry 2007;16:473-483.

- 8 Hubel R, Jass J, Marcus A, Laessle RG: Overweight and basal metabolic rate in boys with attention-deficit/ hyperactivity disorder. Eat Weight Disord 2006;11:139-146.

9 Agranat-Meged AN, Deitcher C, Goldzweig G, Leibenson L, Stein M, Galili-Weisstub E: Childhood obesity and attention deficit/hyperactivity disorder: a newly described comorbidity in obese hospitalized children. Int J Eat Disord 2005;37:357-359.

10 Curtin C, Bandini LG, Perrin EC, Tybor DJ, Must A: Prevalence of overweight in children and adolescents with attention deficit hyperactivity disorder and autism spectrum disorders: a chart review. BMC Pediatr 2005;5:48.

11 Ptacek R, Kuzelova H, Paclt I, Zukov I, Fischer S: Anthropometric changes in non-medicated ADHD boys. Neuro Endocrinol Lett 2009;30:377-381.

12 Nederkoorn C, Braet C, Van Eijs Y, Tanghe A, Jansen A: Why obese children cannot resist food: the role of impulsivity. Eat Behav. 2006; 7:315-322. 
van Egmond-Froehlich et al.: The Hyperactivity/Inattention Subscale of the

Strengths and Difficulties Questionnaire Predicts Short- and Long-Term Weight Loss in Overweight Children and Adolescents Treated as Outpatients

$\$ 13$ Waring ME, Lapane KL: Overweight in children and adolescents in relation to attention-deficit/hyperactivity disorder: results from a national sample. Pediatrics 2008;122:e1-6.

14 Lam LT, Yang L: Overweight/obesity and attention deficit and hyperactivity disorder tendency among adolescents in China. Int J Obes (Lond) 2007;31:584-590.

-15 Erhart M, Herpertz-Dahlmann B, Wille N, Sawitzky-Rose B, Holling H, Ravens-Sieberer U: Examining the relationship between attention-deficit/hyperactivity disorder and overweight in children and adolescents. Eur Child Adolesc Psychiatry 2012;21:39-49. .

16 Drukker M, Wojciechowski F, Feron FJ, Mengelers R, Van Os J: A community study of psychosocial functioning and weight in young children and adolescents. Int J Pediatr Obes 2009;4:91-97.

17 Rojo L, Ruiz E, Dominguez JA, Calaf M, Livianos L: Comorbidity between obesity and attention deficit/ hyperactivity disorder: population study with 13-15-year-olds. Int J Eat Disord 2006;39:519-522.

18 van Egmond-Froehlich AW, Widhalm K, de Zwaan M: Association of symptoms of attention-deficit/hyperactivity disorder with child hood overweight adjusted for confounding parental variables. Int J Obes (Lond) 2012;36:963-968.

19 Graziano PA, Calkins SD, Keane SP: Toddler self-regulation skills predict risk for pediatric obesity. Int J Obes (Lond) 2010;34:633-641.

20 Mustillo S, Worthman C, Erkanli A, Keeler G, Angold A, Costello EJ: Obesity and psychiatric disorder: developmental trajectories. Pediatrics 2003;111(:851-859.

-21 de Zwaan M, Gruss B, Muller A, Philipsen A, Graap H, Martin A, Glaesmer H, Hilbert A: Association between obesity and adult attention-deficit/hyperactivity disorder in a German community-based sample. Obes Facts 2011;4:204-211.

-22 Pagoto SL, Curtin C, Lemon SC, Bandini LG, Schneider KL, Bodenlos JS, Ma Y: Association between adult attention deficit/hyperactivity disorder and obesity in the US population. Obesity (Silver Spring) 2009;17: 539-544.

23 Nederkoorn C, Jansen E, Mulkens S, Jansen A: Impulsivity predicts treatment outcome in obese children. Behav Res Ther 2006;45:1071-1075.

24 Pauli-Pott U, Albayrak O, Hebebrand J, Pott W: Does inhibitory control capacity in overweight and obese children and adolescents predict success in a weight-reduction program? Eur Child Adolesc Psychiatry 2010;19:135-141.

25 Logan GD, Schachar RJ, Tannock R: Impulsivity and inhibitory control. Psychol Sci 1997;8:60-64.

-26 Reinehr T, Hoffmeister U, Mann R, Goldapp C, Westenhofer J, Egmond-Froehlich A, Bullinger M, RavensSieberer U, Holl RW: Medical care of overweight children under real-life conditions: the German BZgA observation study. Int J Obes (Lond) 2009;33:418-423.

27 Cole TJ: The LMS method for constructing normalized growth standards. Eur J Clin Nutr 1990;44:45-60.

-28 Lange M, Kamtsiuris P, Lange C, Schaffrath Rosario A, Stolzenberg H, Lampert T: Sociodemographic characteristics in the German Health Interview and Examination Survey for Children and Adolescents (KiGGS) - operationalisation and public health significance, taking as an example the assessment of general state of health (in German). Bundesgesundheitsblatt Gesundheitsforschung Gesundheitsschutz 2007;50:578589.

-29 Goodman R: Psychometric properties of the strengths and difficulties questionnaire. J Am Acad Child Adolesc Psychiatry 2001;40:1337-1345.

30 Goodman R, Meltzer H, Bailey V: The strengths and difficulties questionnaire: a pilot study on the validity of the self-report version. Int Rev Psychiatry 2003;15:173-177.

31 American Psychiatric Association: Diagnostic and Statistical Manual of Mental Disorders (DSM-IV). Washington, D.C., American Psychiatric Association, 1994.

-32 Goodman R, Scott S: Comparing the Strengths and Difficulties Questionnaire and the Child Behavior Checklist: is small beautiful? J Abnorm Child Psychol 1999;27:17-24.

-33 Hölling H, Erhart M, Ravens-Sieberer U, Schlack R: Behavioural problems in children and adolescents. First results from the German Health Interview and Examination Survey for Children and Adolescents (KiGGS) (in German). Bundesgesundheitsblatt Gesundheitsforschung Gesundheitsschutz 2007;50(5-6):784-93.

34 Goodman R, Ford T, Simmons H, Gatward R, Meltzer H: Using the Strengths and Difficulties Questionnaire (SDQ) to screen for child psychiatric disorders in a community sample. Br J Psychiatry 2000;177:534-539.

35 Westenhöfer J (ed): K-FEV Fragebogen zum Essverhalten für Kinder und Jugendliche. Göttingen, Hogrefe, 2011.

36 Pudel V, Westenhöfer J: Fragebogen zum Essverhalten, Handanweisung. Göttingen, Hogrefe, 1989.

-37 Stunkard AJ, Messick S: The three-factor eating questionnaire to measure dietary restraint, disinhibition and hunger. J Psychosom Res 1985;29:71-83.

-38 White IR, Horton NJ, Carpenter J, Pocock SJ: Strategy for intention to treat analysis in randomised trials with missing outcome data. BMJ 2011;342:d40.

-39 Lumeng JC, Gannon K, Cabral HJ, Frank DA, Zuckerman B: Association between clinically meaningful behavior problems and overweight in children. Pediatrics 2003;112:1138-1145.

40 Hays NP, Roberts SB: Aspects of eating behaviors 'disinhibition' and 'restraint' are related to weight gain and BMI in women. Obesity (Silver Spring) 2008;16:52-58.

41 Savage JS, Hoffman L, Birch LL: Dieting, restraint, and disinhibition predict women's weight change over 6 y. Am J Clin Nutr 2009;90:33-40. 
-42 Sabin MA, Ford A, Hunt L, Jamal R, Crowne EC, Shield JP: Which factors are associated with a successful outcome in a weight management programme for obese children? J Eval Clin Pract 2007;13:364-368.

43 Gasparrini E, Rondina C, Pianelli G, Fabiani E, D’Angelo G, Catassi C, Coppa GV: Treatment of childhood obesity. Long-term outcome and predictive factors of success in a group of 130 subjects in Italian. Minerva Pediatr 2003;55:33-41.

\$4 Eliakim A, Friedland O, Kowen G, Wolach B, Nemet D: Parental obesity and higher pre-intervention BMI reduce the likelihood of a multidisciplinary childhood obesity program to succeed - a clinical observation. J Pediatr Endocrinol Metab 2004;17:1055-1061.

-45 Bohler T, Alex C, Becker E, Becker R, Hoffmann S, Hutzler D, Jung C, Laufersweiler-Lochmann F, Radu C: Quality indicators for ambulatory health education programme for overweight and obese children and adolescents (in German). Gesundheitswesen 2004;66:748-753.

-46 Pagoto SL, Curtin C, Bandini LG, Anderson SE, Schneider KL, Bodenlos JS, Ma Y: Weight loss following a clinic-based weight loss program among adults with attention deficit/hyperactivity disorder symptoms. Eat Weight Disord 2010;15:e166-172.

47 Golan M, Kaufman V, Shahar DR: Childhood obesity treatment: targeting parents exclusively v. parents and children. Br J Nutr 2006;95:1008-1015.

-48 Uzark KC, Becker MH, Dielman TE, Rocchini AP, Katch V: Perceptions held by obese children and their parents: implications for weight control intervention. Health Educ Q 1988;15:185-198.

49 Reinehr T, Brylak K, Alexy U, Kersting M, Andler W: Predictors to success in outpatient training in obese children and adolescents. Int J Obes Relat Metab Disord 2003;27:1087-1092.

50 Epstein LH, Wisniewski L, Weng R: Child and parent psychological problems influence child weight control. Obes Res 1994;2:509-515.

-51 Braet C: Patient characteristics as predictors of weight loss after an obesity treatment for children. Obesity (Silver Spring) 2006;14:148-155.

-52 Goodman A, Goodman R: Strengths and difficulties questionnaire as a dimensional measure of child mental health. J Am Acad Child Adolesc Psychiatry 2009;48:400-403.

53 Becker A, Hagenberg N, Roessner V, Woerner W, Rothenberger A: Evaluation of the self-reported SDQ in a clinical setting: do self-reports tell us more than ratings by adult informants? Eur Child Adolesc Psychiatry 2004;13(suppl 2):II17-24. 